\title{
IoT Based Green House Monitoring Using Data Compressive Sensing Technique in WSN

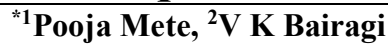 \\ ${ }^{1,2}$ Department of E\&TC, AISSMS Institute of Information Technology, Pune, India Email:poojamete2013@gmail.com,vbairagi@yahoo.co.in
}

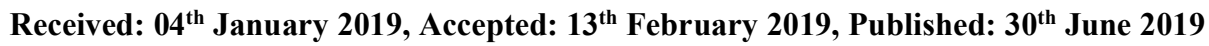

\begin{abstract}
Compressive Sampling is one of the important techniques for energy proficient transmission in wireless sensor. It is also utilized for low-sampling in several applications. Compressive sensing may reduce energy cost when sensor development is taken in to consideration. Green house farming is need of hour. Efficient management of resources is vital. An IOT based green house monitoring is intended to screen and control the different elements received from a farm field, like stickiness, water level and temperature. The data is gathered through wireless sensor network. Large data is generated through different sensors which required considerable storage space and increase transmission time over network from slave to master. Compression is effective in compressing such data. In this paper, we propose an energy effective transmission approach based on compression sensing for WSN.
\end{abstract}

Keywords

Compressive Sensing; WSN, Green House Monitoring, Internet of Things.

\section{Introduction}

An Internet of Things (IOT) Based green house framework is intended to screen and control the different data components received from a rural field, for example, dampness, water level, temperature, and human association. This framework is large and made out many of controllers and remote sensors organized in special way. It is utilizing RF Module as the transmission element for identifying estimations of a horticultural field. The sensor assembles different farming elements progressively; utilizing Internet of Things applications. Framework is intended to screen and control the stickiness, water level, temperature, and human association. This framework's most part made up of controllers and a remote sensor.

The sensors assembles different agrarian factors continuously and transmit those to central location (may be server) by utilizing Internet of Thing (IoT) applications through wireless sensor network (WSN). The water system administration framework is one of the approaches to enhance the water system procedures to upgrade the utilization of water, power utilization and work costs. In such manner, with the new innovation and the improvement of the Internet and the Internet of Things, a system for water irrigation system in Indian districts in view of the utilization of the Internet of Things and new correspondence advances is proposed. In this paper, new method proposed for smart water system utilizing IoT. This new component enable to the big screen and oversee horticultural territory utilizing advanced mobile phones through Internet [1].

An information gathering is vital task for adaptability of system. One must take care of reducing and controlling the information loss because of limit of sensor nodes. Data compression is utilized to manage energy effectiveness. Relating above limitation on sources, for example, energy, memory, computational speed and correspondence data IOT based Green House Monitoring Using Data Compressive Protocol transfer capacity brings about Size and cost constraints on sensor node [2].

\section{Compressive Sensing Techniques}

There are two types of data collection techniques, like network based and compression based. The objective of data collection technique is to collect data from various sensors.

A) Compression based technique is utilized to expand the memory and to save the energy of system. It centers on preservation and prolongation of system lifetime. There are different types of compression which are discussed below.

- Compressive Sensing: This is a most proposed and utilized methodology in data gathering process. The focal focus of this technique is energy capability. The signal is compressed at the time of acquisition only [3].

- Conventional Compression: In this method, the information is compacted at the source and decompressed at the destination. This requires more correspondence among the sensor nodes [4] [5].

B) Network based technique: The network lifetime which focuses on maximizing this technique. It is mainly divided into two main parts.

- $\quad$ Routing based: The basic point of this strategy is to pick up the suitable and proficient route and way to assemble information to achieve goal with irrelevant utilization of energy by sensor node. This procedure is proposed to center around transmission power and stream of system [7]. 
- Network coding based: This is sensible approach of data transmission, where the nodes reproduces and progresses the moving toward data package to the center node ultimately accomplishes objective. This procedure improves the network capacity [7].

Performance Analysis Using Compressive Sensing

Lets consider the network of $\mathrm{n}$ ( 6 in present case) nodes. Each node acquires sample di, (at time instant) such as temperature, humidity, and soil moisture.

As seen in figure 1, the routing topology forms data gathering and routing tree. It collects data at the sink node. In compressive sensing, sampled data is transmitted from leaf node to root node in sequence. Fig .1 shows the data gathering with compressive sensing by six nodes.

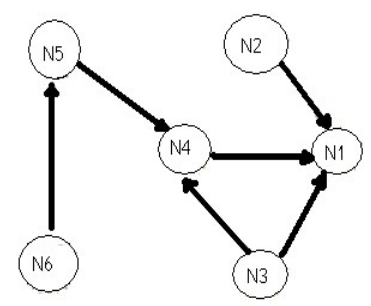

Figure 1: Data Gathering with Compressive Sensing by Nodes

Finally $\mathrm{N}_{1}$ receives the compressed data,

$\sum_{j=1}^{\mathrm{5}} A_{i, j} d_{j}$

and forwards it to the sink.

\section{Proposed System}

In present work, we deal with efficient transmission approach and extensive experiments to verify the superiority of compressive sensing. The wireless sensor network consists of node group which is connected to base station. Environmental condition like temperature, pressure, humidity, pollution and many more, pass that data to other location by similar network. Several activities, such as tracking targets, collecting and storing data, are used in many wireless applications. Each sensor will select the data using less power before sending the data packets again. The data packet size is used as a comparison metric. At the beginning, the leaf node sends its samples adopting the traditional scheme. The reason is that the forwarding cost is lower than the transmission cost of the requested measures.

An automated irrigation system framework is planned to screen and control the different components got from an agricultural field, for e.g. humidity, water level, temperature, and human association. The most part of this framework is made out of controllers and a remote sensor for an agricultural field.

The sensor assembles the different real time components progressively and transmits those utilizing Internet of Thing (IoT) applications. The joining of present day innovation in irrigation management system is one of the best approaches to upgrade procedures of water systems to enhance the utilization of water, power utilization and work costs. In this way, with the new innovation and the advancement of the Internet and the Internet of Things, we proposed a procedure for smart irrigation in Indian regions.

Here we proposed new plan for intelligent irrigation utilizing IoT. This new plan enables the farmer to monitor and oversee agriculture area utilizing PDAs by means of Internet. As discussed in above section, the present system has a wireless network that will work using data compression protocol, as well as sending cumulative data in IOT using an Android application. Then the user can analyze the data in the APPLICATION anywhere. In above system, WSN are reconfigured using RF communication. Here we have considered 1 Master and 2 slave's structure as shown in figure 2. In this network, we are using the data compression protocol. Slave is communicated with master. If all the slaves are transmitting the data to Master then the whole network will be flooded with data frames causing congestion. The data congestion will further reduces the overall efficiency of the WSN. To overcome these limitations, a request and respond protocol are used. The slaves transmit their data frames to their nearest neighbor which is closer to the master.

There will be data joining process at nodes. Next frame is generated by old frame. There will be identifies from sensors. In this way, the data is sent to the master without any collision as the frame is forwarded in the network until finally it reaches the destination (Master).

Data frame: (Existing system)

SOF(Start of frame), Destination ID, Transmitter ID, R, (Data), EOF(End of frame)

Data frame: (Proposed system)

SOF(Start of frame), Destination ID ,Transmitter ID1, (Data), Transmitter ID2, (Data), Transmitter ID3, (Data) ,..... EOF

Therefore, when the compressed data frame finally arrives at the master, the master will separate the data from the frame using the slave IDs (transmitter ID in the previous frame). 


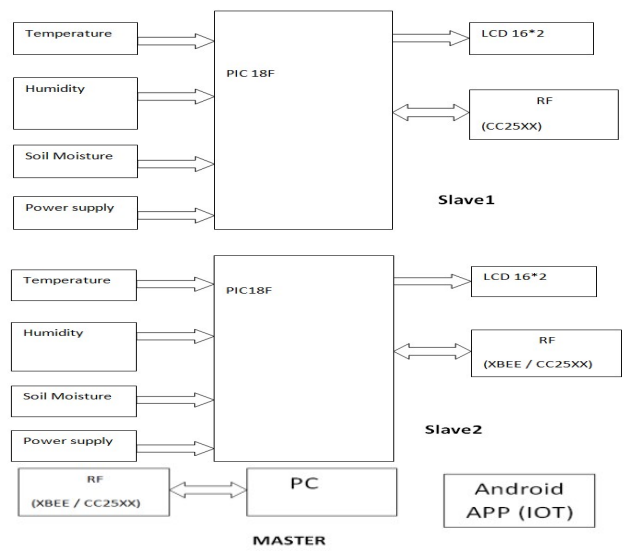

Figure 2: Block Diagram

Visual Basic software is used further in which GUI is developed that will show all the parameters, tables and graphic formats.

\section{Compressive Data Sensing Protocol}

Here we are implementing the Compressive data sensing protocol. Many applications in WSN have big chunks of data, this data needs to be transmitted to the sink node as efficiently as possible. As the number of slaves increase or the sensors increase the data gathered by slaves keeps on increasing. As the slaves are interfaced to Embedded controller, whose data storage capacity is low. So, this data cannot be stored on board the slaves and has to be transmitted to the sink over WSN.

In any normal wireless senor network the data flows either through slaves to master (ADODV protocol) or master (sink) sends a continuous request to slaves and slaves then respond with the data information (Leach / Request and response protocol). In the later method the sink node while sending the request frame includes the destination ID. All slaves receive the request and compare the destination ID to their own ID. If it matches then the data is sent back to the sink node.

In REQ and RES method the sink node continuously sends the request to all the saves present in the WSN. This causes huge amounts of data flow throughout the network. Most of the times the data transmissions causes network congestion reducing network efficiency.

The main Idea of compressed data algorithm is that we divide the whole WSN in group of clusters. Each cluster is having a cluster head which will gather the data generated within the cluster. Here the slaves within the cluster transfer their data through REQ and RES protocol. The cluster head will then compress all the data frames sent by slaves into a single frame and then send it to Master (Sink) Node. Due to this there are lesser data to gather in WSN.

Since the cluster head is compressing the data of all sensors in one frame, separate data transmissions are not required which reduces the overall load of network which makes it congestion free and increases the network efficiency.

All the slaves are connected to sensors which perform the task of continuous sensing. In most of the applications local conditions do not change over a long periods of time. So, sensing the same data over and over again, results in less network life. In our algorithm the slave will transmit its own data whenever any sensor has crossed its set point. If the data sensed by microcontroller is found same as the previous data then it becomes redundant.

Whenever there is critical event occurring the corresponding slave transmits the data to the Cluster head node instantly. The communication must continues until the critical event has over.

\section{Algorithm \\ Data Compression}

A Compression algorithms that uses arithmetic encoding begin by determining a data model, basically a prediction of which models will be found in the message symbols. The more accurate this forecast is, the closer the optimal output will be.

Algorithm:

\section{Cluster head}

Start

Init LCD and other hardware

"A"

Send request to Slave

Is response received? 
If, No $\rightarrow$ Send own frame to Master

If, $\mathrm{No} \rightarrow$ "B"

"B"

If, $\mathrm{Yes} \rightarrow$ Send the compressed frame to master.

Read sensors through ADC

Store and display sensor data on LCD $\rightarrow$ "A"

Slave

Start

Init LCD and other hardware

"A"

Is response received?

If, No $\rightarrow$ "B"

If, Yes $\rightarrow$ Is data redundant?

No $\rightarrow$ Send the own frame to master

"B"

Read sensors through ADC

Store and display sensor data on LCD $\rightarrow$ "A"

Implementation and Results

Here we are going to demonstrate the data in a graphical format through which the user can monitor the data in real time. Likewise we propose the data information in a graphical format with the goal that the data compared in real time.

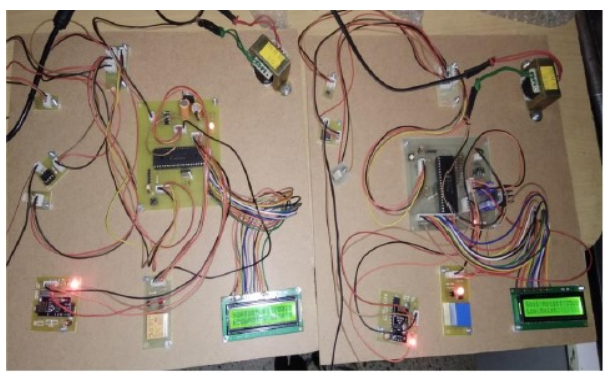

Iot O/P Result:

Figure 3: Actual Experimental Setup with Master and Slave

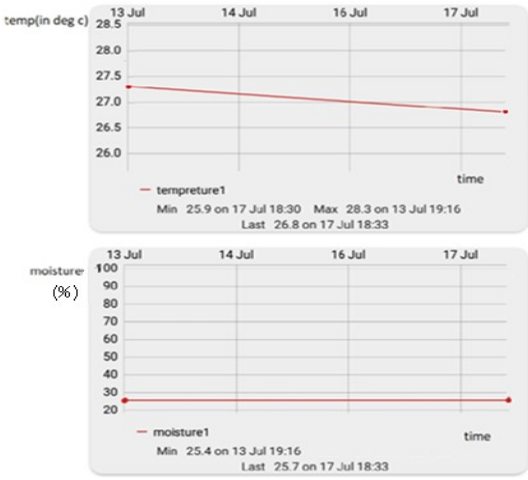

Figure 4: Graphs of Temperature 1 and Moisture1 on IoT

Above figure illustrate graphical readings of tempreature1 and moisture1 according to visual basic gives data. These graphical readings are shown on IoT application.

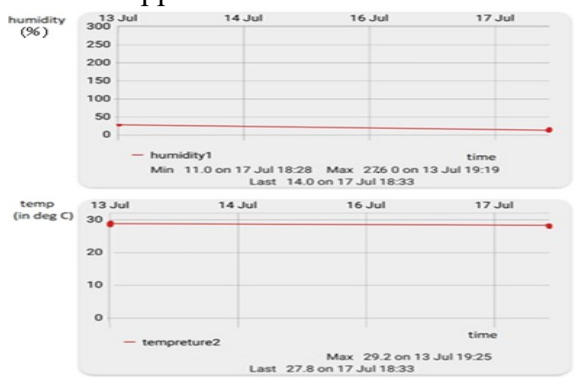

Figure 5: Graphs of Humidity 1 and Temperature 2 on IoT 
Above figure illustrate graphical readings of humidity1 and tempreture 2 according to visual basic gives data. These graphical readings are shown on IoT application.

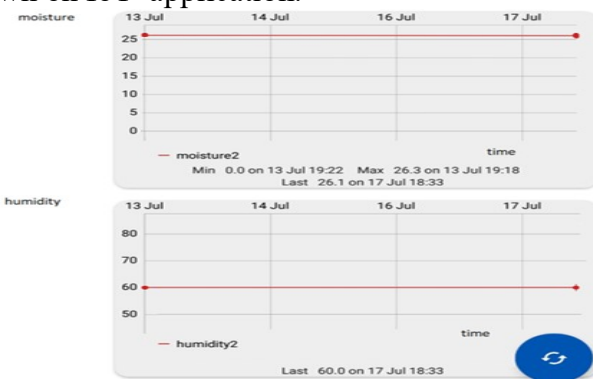

Figure 6: Graphs of Moisture 2 and Humidity 2 on IoT

Above figure illustrate graphical readings of soil moisture 2 and humidity 2 according to visual basic gives data. These graphical readings are shown on IoT application.

VB Result

${ }^{0} \mathbf{c}, \%, \%$

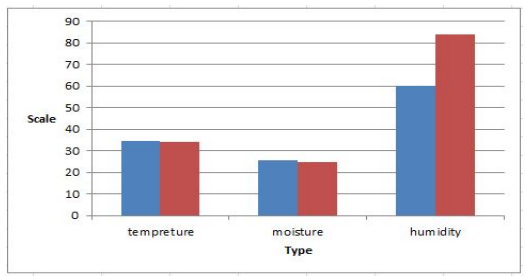

Time

Figure 7: Bar chart 1:Compressed Frame of Cluster and Slave Data

Above graph shows compressed frame of cluster and slave data which gives all the readings of temperature, soil moisture and humidity at a particular time period. It takes data from Graphical user interface (GUI) developed by visual basic which receive data sent by greenhouse and also save it to internal database. There will not be any data on visual basic. So both cluster and slave are in redundant mode

${ }^{0} \mathbf{c}, \%, \%$

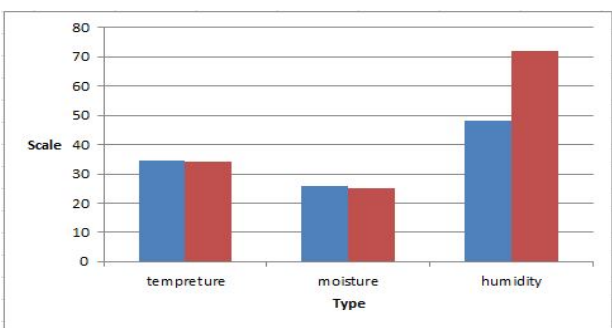

Time

Figure 8: Bar Chart 2 :Non Compressed, Only Slave Data Cluster is in Redundant Mode

Above figure shows only slave data, because in cluster if soil moisture increases remaining temperature and humidity can not shown at a particular time period. So the complete cluster is in redundant mode. But it doesn't affect on slave and continuously takes reading according to time, therefore it is also non compressed .

\section{Comparison Table}

\begin{tabular}{|c|c|c|}
\hline Sensors & Values & Time \\
\hline Temperature1 & 34.5 & 14.52 \\
\hline Moisture1 & 25.8 & 14.52 \\
\hline Humidity1 & 60.0 & 14.52 \\
\hline Temperature2 & 34.0 & 14.52 \\
\hline Moisture2 & 95.0 & 14.54 \\
\hline Humidity2 & 72.0 & 14.54 \\
\hline
\end{tabular}

Table 1: Values of Temperature, Moisture, Humidity on different IOT 
This table shows readings about sensor values and time at a particular mode respectivley. It gives different values and time according to tempreture humidity and soil moisture on Iot app. So that it is easy to monitoring. This experiment is just tested for its working in lab environment. The circuit needs to be calibrated for actual use in green house. The sensors needs to be calibrated to match actual vaules.

\section{Obeservation and Readings}

- At normal codition data calculated,

Master count rate $=1 \mathrm{sec}$

In $1 \mathrm{hr}$, count $=3600$

Normal end response for 1 count $=28$ bytes

$1 \mathrm{hr}=28 * 3600=100800$ bytes

- After Condition changes,

Response only when data changes,

If slave 1 data change in $1 \mathrm{hr}=60$

$1 \mathrm{hr}$ data $=28 * 60=1680$ bytes

So, data compressed is $=99120$ bytes.

\section{Conclusions \& Research Challenges}

In this system, wireless sensor network for green house monitoring application for energy efficient transmission approach using data aggregation is discussed and implemented. Compressive Sensing algorithms are developed for providing the improvement in utilization of limited resources of WSN. The implemented circuit needs calibration for actual real times reading in greenhouse. In future, development of an IOT based application can be proposed through which the user can monitor parameters remotely. One can also work on an algorithm for anti-collision to reduce congestion and increase lifetime.

\section{References}

[1] Z. Li, J. Wang, R. Higgs, L. Zhou and W. Yuan, "Design of an Intelligent Management System for Agricultural Greenhouses Based on the Internet of Things," 2017 IEEE International Conference on Computational Science and Engineering (CSE) and IEEE International Conference on Embedded and Ubiquitous Computing (EUC), Guangzhou, 2017, 154-160.

[2] H. Yang, K. Tang, H. Xu and X. Wang, "Energy-Efficient Transmission Based on Compressive Sensing in WSN," 2017 IEEE Wireless Communications and Networking Conference (WCNC), San Francisco, CA, 2017, $1-6$.

[3] Nikhil S. Hage, S. Kayalvizhi, "Efficient Data Gathering with Compressive Sensing in Wireless Sensor Networks", International Research Journal of Engineering and Technology (IRJET), Vol: 03 No 01, Jan-2016.

[4] Razzaque, M.A.; Bleakley, C.; Dobson, S. Compression in wireless sensor networks: A survey and comparative evaluation. ACM Trans. Sens. Netw. 2013, 10, 5:1-5:44.

[5] Xiao-Yang Liu, Yanmin Zhu, Linghe Kong, Cong Liu, Yu Gu, Athanasios V. Vasilakos, Min-You Wu, "CDC: Compressive Data Collection for Wireless Sensor Networks," in IEEE Transactions on Parallel and Distributed Systems, vol. 26, no. 8, pp. 2188-2197, 1 Aug. 2015

[6] Giuseppe Campobello, Antonino Segreto, and Salvatore Serrano, "Data Gathering Techniques for Wireless Sensor Networks: A Comparison", in Proc. International Journal of Distributed Sensor Networks, Vol 2016, Feb 2016.

[7] A. Masoum, N. Meratnia and P. J. M.Havinga, "A distributed compressive sensing technique for data gathering in wireless sensor networks," Procedia computer Science, vol. 21, pp.207-216, Sep. 2013.

[8] Yanjun Yao, Qing Cao, Athanasios V. Vasilakos, "EDAL: an Energy efficient, Delay-aware, and Lifetimebalancing Data Collection Protocol for Heterogeneous Wireless Sensor Networks", in Proc. National Science Foundation grant CNS-0953238, CNS-1017156, CNS-1117384, and CNS-1239478.

[9] Luo, C.; Wu, F.; Sun, J.; Chen, C. Efficient measurement generation and pervasive sparsity for compressive data gathering. IEEE Trans. Wirel. Commun. 2010, 9, 3728-3738.

[10] Chou, C.T.; Rana, R.K.; Hu, W. Energy Efficient Information Collection in Wireless Sensor Networks Using Adaptive Compressive Sensing. In Proceedings of IEEE 34th Conference on Local Computer Networks, Zurich, Switzerland, 20-23 October 2009; pp. 443-450. 